\title{
Exploration of jet energy loss via direct $\gamma$-charged particle azimuthal correlation measurements
}

\author{
A. M. Hamed for the STAR collaboration \\ Cyclotron Institute, Texas AEM University, College Station, TX 77843, USA
}

\begin{abstract}
The multiplicities of charged particles azimuthally associated with direct photons and $\pi^{0}$ have been measured for $\mathrm{Au}+\mathrm{Au}, \mathrm{p}+\mathrm{p}$, and $\mathrm{d}+\mathrm{Au}$ collisions at $\sqrt{s_{N N}}=200 \mathrm{GeV}$ in the STAR experiment. Charged particles with transverse momentum $0.5<p_{T}^{h^{ \pm}}<16 \mathrm{GeV} / \mathrm{c}$ for $\mathrm{p}+\mathrm{p}$ and $\mathrm{d}+\mathrm{Au}$, and $3<p_{T}^{h^{ \pm}}<16 \mathrm{GeV} / \mathrm{c}$ for $\mathrm{Au}+\mathrm{Au}$ and pseudorapidity $|\eta| \leq 1.5$ in coincidence with direct photons and $\pi^{0}$ of high transverse momentum $8<p_{T}^{\gamma, \pi^{0}}<16 \mathrm{GeV} / \mathrm{c}$ at $|\eta| \leq 0.9$ have been used for this analysis. Within the considered range of kinematics, the observed suppressions of the associated yields per direct $\gamma$ in central $\mathrm{Au}+\mathrm{Au}$ relative to $\mathrm{p}+\mathrm{p}$ and $\mathrm{d}+\mathrm{Au}$ are similar and constant with direct photon fractional energy $z_{T}\left(z_{T}=p_{T}^{h^{ \pm}} / p_{T}^{\gamma}\right)$. The measured suppressions of the associated yields with direct $\gamma$ are comparable to those with $\pi^{0}$. The data are compared to theoretical predictions.
\end{abstract}

In inverse Compton scattering $(q+g \rightarrow q+\gamma)$ and quark annihilation $(q+\bar{q} \rightarrow g+\gamma)$ the transverse momentum of the outgoing high- $p_{T} \gamma$ counterbalances the transverse momentum of the outgoing parton, modulo negligible corrections from initial-state radiation. Therefore, direct $\gamma$-hadron azimuthal correlations have been proposed as a good probe for the jet energy loss, especially its dependence on the parton initial energy, in the medium created at relativistic heavy ion collisions [1]. Moreover, in heavy ion collisions, the produced $\gamma$ departs the medium upon creation without any further interactions, and therefore doesn't experience any geometrical bias which might arise from the hard-scattering vertex position inside the medium. Accordingly, a comparison of direct $\gamma$-hadron azimuthal correlations with di-hadron azimuthal correlations could provide additional constraints on the path-length dependence of energy loss. We report on the results of $\gamma$-charged particles $(\gamma$-ch $)$ and $\pi^{0}$-charged particles $\left(\pi^{0}\right.$-ch) azimuthal correlation measurements in the STAR experiment.

The most challenging aspect of this analysis is to separate the direct photons from background photons. In fact the remnants of any source of background could obscure the parton initial energy, and cause the $\gamma$-jet coincidence measurements to suffer from the same geometrical biases as of di-hadron analysis. Among the hadronic decays, $\pi^{0}$ and $\eta$ are the main known sources of direct photon backgrounds in hadronic and nuclear collisions. The STAR experiment at RHIC is very well adapted for such challenge through its Barrel Electromagnetic Calorimeter (BEMC) [2]. Utilizing the transverse profile technique of electromagnetic showers in the STAR Shower Maximum Detector with its fine segmentation $(\Delta \eta \approx 0.007, \Delta \phi \approx 0.007)$, discrimination between the $(2 \gamma) /(1 \gamma)$ showers up to $p_{T}^{\pi^{0}} \sim 26 \mathrm{GeV} / \mathrm{c}$ becomes possible. Furthermore, the BEMC tower size $(\Delta \eta=0.05, \Delta \phi=0.05)$ is typically greater than the angular separation between the two 


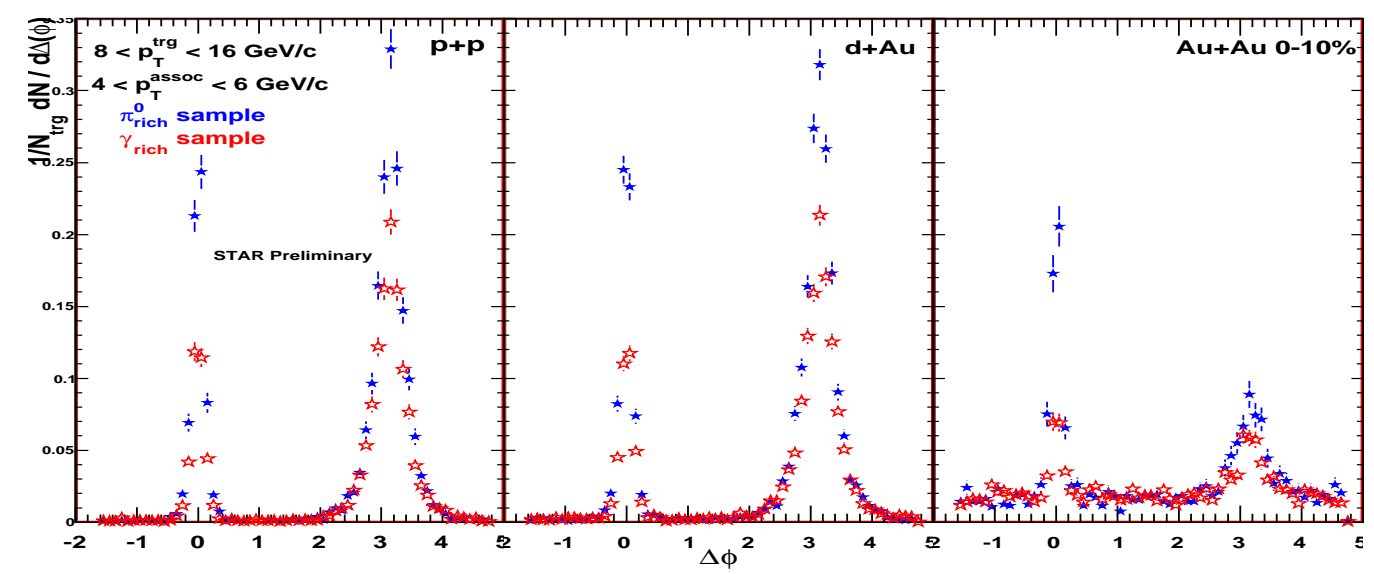

Figure 1: Azimuthal correlation histograms of $\gamma_{\text {rich }}$ sample and $\pi_{\text {rich }}^{0}$ sample $8.0<p_{T}^{\gamma, \pi^{0}}<16.0 \mathrm{GeV} / \mathrm{c}$ at $|\eta| \leq 0.9$ with associated charged particles $4.0<p_{T}^{h^{ \pm}}<6.0 \mathrm{GeV} / \mathrm{c}$ at $|\eta| \leq 1.5$ for $\mathrm{p}+\mathrm{p}$, d+Au and $0-10 \% \mathrm{Au}+\mathrm{Au}$ collisions at $\sqrt{s_{N N}}$ $=200 \mathrm{GeV}$.

photons resulting from a symmetric decay of high- $p_{T} \pi^{0}$. Therefore, the determination of $\pi^{0} \mathrm{~s}$ $p_{T}$, without invariant mass reconstruction of $\pi^{0}$, is attainable.

The STAR detector is very well-suited for azimuthal correlations due to the full coverage in azimuth of the BEMC and the STAR main Time Projection Chamber (TPC) [3]. Using a level-2 high- $E_{T}$ tower trigger to tag $\gamma$-jet events, in 2007 the STAR experiment collected an integrated luminosity of $535 \mu \mathrm{b}^{-1}$ of $\mathrm{Au}+\mathrm{Au}$ collisions at $\sqrt{s_{N N}}=200 \mathrm{GeV}$. As a reference measurement, the $\mathrm{p}+\mathrm{p}$ data taken in 2006 with integrated luminosity of $11 \mathrm{pb}^{-1}$, and $34 \mathrm{nb}^{-1}$ of $\mathrm{d}+\mathrm{Au}$ data taken in 2008 at $\sqrt{s_{N N}}=200 \mathrm{GeV}$ are analyzed. In this analysis the $\pi^{0} / \gamma$ discrimination was carried out by making cuts on the shower shape, where the $\pi^{0}$ identification cut was adjusted in order to obtain a direct $\gamma$ free $\left(\pi_{\text {rich }}^{0}\right)$ sample and a sample rich in direct $\gamma\left(\gamma_{\text {rich }}\right)$.

Figure 1 shows the azimuthal correlation for $\gamma_{\text {rich }}$ sample triggers and $\pi_{\text {rich }}^{0}$ triggers for $\mathrm{p}+\mathrm{p}$, $\mathrm{d}+\mathrm{Au}$, and $\mathrm{Au}+\mathrm{Au} 0-10 \%$. The medium effect is obviously seen in the away side as it was previously reported [4]. As predicted, the $\gamma_{\text {rich }}$ triggered sample has lower near-side yields than these of the $\pi_{\text {rich }}^{0}$ but not zero. The non-zero near-side yield for the $\gamma_{\text {rich }}$ sample is expected due to the remaining contributions of the widely separated photons from other sources. It is noticeable that the ratio of the away side to near side yields of the $\gamma_{\text {rich }}$ sample is slightly greater than that of $\pi_{\text {rich }}^{0}$ sample which might be due to the effect of direct $\gamma$.

In order to determine the combinatorial background level, the relative azimuthal angular distribution of the associated particles with respect to the trigger particle is simply fitted with two Gaussian peaks and a straight line. The near- and away-side yields, $Y^{n}$ and $Y^{a}$, of associated particles per trigger are extracted by integrating the $1 / N_{\text {trig }} d N / d(\Delta \phi)$ distributions in $|\Delta \phi| \leq 0.63$ and $|\Delta \phi-\pi| \leq 0.63$ respectively. Although as discussed later, the purity of the $\pi_{\text {rich }}^{0}$ sample is not critical for this analysis as long as $\pi_{\text {rich }}^{0}$ sample is free of direct $\gamma$, a general agreement between the results from this analysis $\left(\pi^{0}\right.$-ch) and the previous analysis (ch-ch) [4] is clearly seen in Fig. 2.(left), which shows the $z_{T}=p_{T}^{\text {assoc }} / p_{T}^{\text {trig }}$ dependence of the associated yield normalized per 

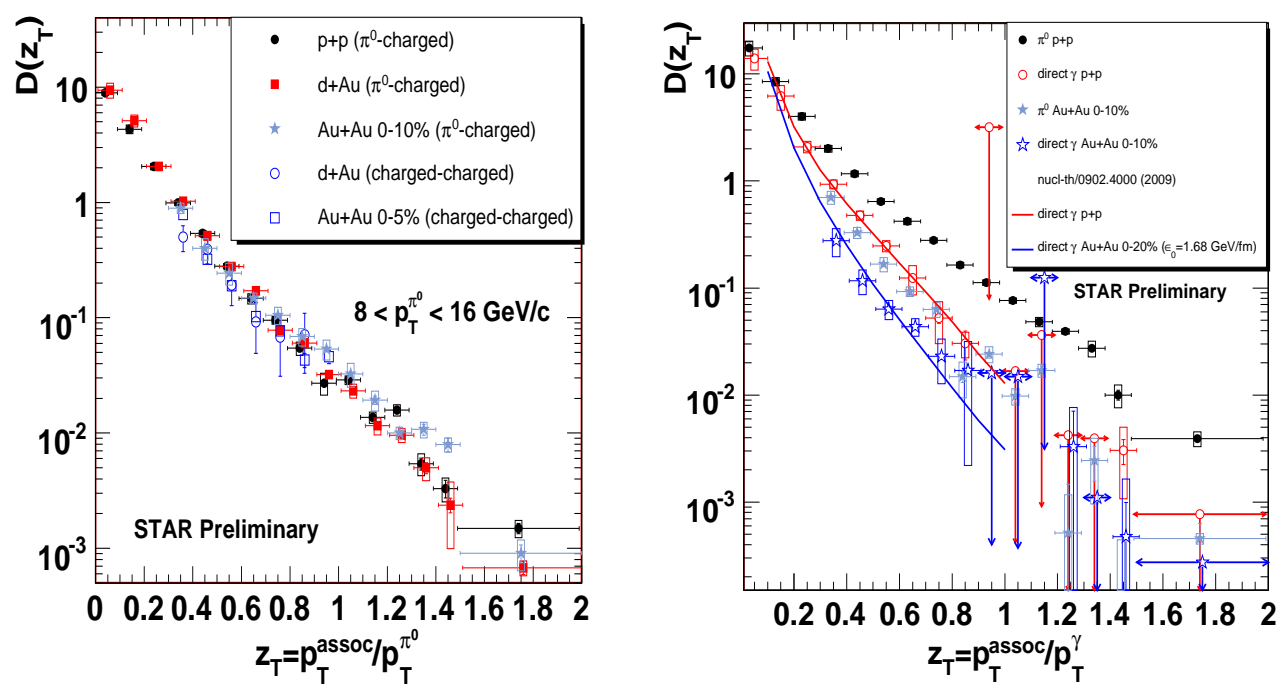

Figure 2: (Left panel) The associated near side yield normalized per hadron trigger, the ch-ch data (open symbols) are from [4]. (Right panel) The associated away side yield normalized per trigger for direct $\gamma$ (open symbols) and $\pi^{0}$ (closed symbols) of $8.0<p_{T}^{\gamma, \pi^{0}}<16.0 \mathrm{GeV} / \mathrm{c}$ for $\mathrm{p}+\mathrm{p}$, and $0-10 \% \mathrm{Au}+\mathrm{Au}$ collisions at $\sqrt{s_{N N}}=200 \mathrm{GeV}$. The upper limits are for $90 \%$ confidence levels. Theoretical curves are within NLO calculations and $\epsilon_{0}$ is the energy loss parameter proportional to initial average gluon density $\rho_{0}[5]$.

trigger $D\left(z_{T}\right)$.

Assuming zero near-side yield for direct photon triggers and a sample of $\pi^{0}$ which is free of direct $\gamma$, the away-side yield of hadrons correlated with the direct photon is extracted as

$$
Y_{\gamma_{\text {direct }}+h}=\frac{Y_{\gamma_{\text {rich }}+h}^{a}-\mathcal{R} Y_{\gamma_{\text {rich }}+h}^{n}}{1-r}
$$

where $\mathcal{R}=Y_{\pi_{\text {rich }}^{0}+h}^{a} / Y_{\pi_{\text {rich }}^{0}+h}^{n}, r=Y_{\gamma_{\text {rich }}+h}^{n} / Y_{\pi_{\text {rich }}^{0}+h}^{n}, 1-r=N^{\gamma_{\text {dir }}} / N^{\gamma_{\text {rich }}}$, and $N^{\gamma_{\text {dir }}}$ and $N^{\gamma_{\text {rich }}}$ are the numbers of triggers of direct $\gamma$ and $\gamma_{\text {rich }}$ respectively. In Eq. 1 all sources of background are approximated to the measured $\pi^{0}$ sample where $\mathcal{R}=Y_{\pi^{0}+h}^{a} / Y_{\pi^{0}+h}^{n}$, but, to the extent that this ratio is similar for all sources of background and/or the fraction of other sources is small, this approximation is valid.

Figure 2 (right) shows the $z_{T}$ dependence of the trigger-normalized fragmentation function for $\mathrm{p}+\mathrm{p}$ and central $\mathrm{Au}+\mathrm{Au}$ 0-10\%. Within the NLO calculations for the associated yield with direct $\gamma$ triggers [5], theory describes the $\mathrm{p}+\mathrm{p}$ and $\mathrm{Au}+\mathrm{Au}$ data within errors. The away-side yield per direct $\gamma$ is lower than that of $\pi^{0}$ for $\mathrm{p}+\mathrm{p}$ collisions as expected, since quarks and gluons are present in different proportions opposite to direct $\gamma$ and $\pi^{0}$ triggers, and $\pi^{0}$ results from higher parton initial energy. It is expected that the away side parton of $\pi^{0}$-ch to travel a longer distance through the medium and lose more energy due to the bi-colored nature of the gluon. However, the results show that the medium has similar effect on the multiplicity of the away-side associated charged particles with direct $\gamma$ and $\pi^{0}$ for central $\mathrm{Au}+\mathrm{Au}$ collisions. 

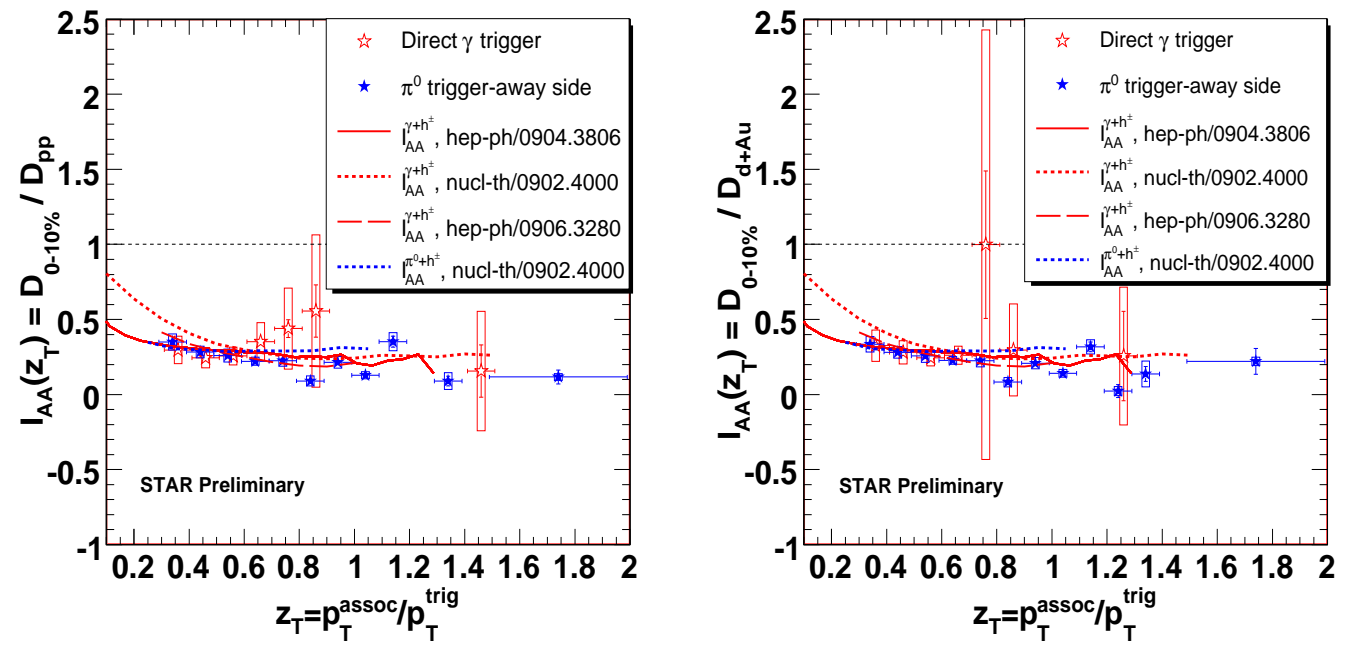

Figure 3: Ratio $I_{A A}$ of $\pi^{0}$ (closed symbols) and direct $\gamma$ (open symbols) of $8.0<p_{T}^{\gamma, \pi^{0}}<16.0 \mathrm{GeV} / \mathrm{c}$ at $\sqrt{s_{N N}}=200$ $\mathrm{GeV}$ relative to $\mathrm{p}+\mathrm{p}$ (left panel) and relative to $\mathrm{d}+\mathrm{Au}$ (right panel). Theoretical predictions are from [5,6,7]. Reference [5] considers the NLO contributions, reference [6] examines schematic conjectures of energy loss, and reference [7] incorporates the complete set of photon production channels.

For better quantification, the ratio $\left(I_{A A}\right)$ of the integrated away-side yield of the associated particles per trigger in $A u+A u$ relative to $p+p$ and relative to $d+A u$ are shown in Fig.3. The similar suppression of the per-trigger away side multiplicity for $\mathrm{Au}+\mathrm{Au}$ with respect to $\mathrm{d}+\mathrm{Au}$ and $p+p$ indicates the final state effect. The suppression of direct $\gamma$ over the shown range of $z_{T}$ doesn't indicate a significant dependence of energy loss on the parton initial energy and might eliminate the conjectured typical energy loss and constant fractional energy loss [6]. While the data are well-described at high $z_{T}$ with different theoretical calculations $[5,6,7]$, results seem to disfavor the volume emission [5] at low $z_{T}$. Despite all the expected differences between the di-hadron and $\gamma$-hadron correlations, the value of $I_{A A}^{\gamma h^{ \pm}}$and $I_{A A}^{\pi^{0} h^{ \pm}}$are similar.

In summary, the energy loss is a final state effect. The constant suppression of $I_{A A}^{\gamma h^{ \pm}}$with direct photon fractional energy may indicate that the energy loss has no strong dependence on the initial parton energy. The similarity of $I_{A A}^{\gamma h^{ \pm}}$and $I_{A A}^{\pi^{0} h^{ \pm}}$indicates either no significant path length dependence of energy loss or the interplay between different factors blurred the expected difference. Further extension to low $z_{T}$ may permit the discrimination between different models.

\section{References}

[1] X.N. Wang, Z. Huang, and I. Sarcevic., Phys. Rev. Lett. 77 (1996).

[2] M. Beddo et al. , Nucl. Instrum. Meth. A 499725 (2003).

[3] M. Anderson et al. , Nucl. Instrum. Meth. A 499659 (2003).

[4] J. Adams et al., Phys. Rev. Lett. 97162301 (2006).

[5] H. Zhang, J.F. Owens, E. Wang, and X.N. Wang, arXiv:nucl-th/0902.4000 (2009).

[6] T. Renk, arXiv:hep-ph/0904.3806 (2009).

[7] G.Y Qin, J. Ruppert, C. Gale, S. Jeon, and G. Moore, arXiv:hep-ph/0906.3280 (2009). 